\title{
Magnetic energy release: flares and coronal mass ejections
}

\author{
Cristina H. Mandrini \\ Instituto de Astronomía y Física del Espacio (CONICET-UBA), \\ CC 67, Suc 28, 1428 Buenos Aires, Argentina \\ e-mail: mandrini@iafe.uba.ar
}

\begin{abstract}
Free energy stored in the magnetic field is the source that powers solar and stellar activity at all temporal and spatial scales. The energy released during transient atmospheric events is contained in current-carrying magnetic fields that have emerged twisted and may be further stressed via motions in the lower atmospheric layers (i.e. loop-footpoint motions). Magnetic reconnection is thought to be the mechanism through which the stored magnetic energy is transformed into kinetic energy of accelerated particles and mass flows, and radiative energy along the whole electromagnetic spectrum. This mechanism works efficiently at scale lengths much below the spatial resolution of even the highest resolution solar instruments; however, it may imply a large-scale restructuring of the magnetic field inferred indirectly from the combined analysis of observations and models of the magnetic field topology. The aftermath of magnetic energy release includes events ranging from nanoflares, which are below our detection limit, to powerful flares, which may be accompanied by the ejection of large amounts of plasma and magnetic field (so called coronal mass ejections, CMEs), depending on the amount of total available free magnetic energy, the magnetic flux density distribution, the magnetic field configuration, etc. We describe key observational signatures of flares and CMEs on the Sun, their magnetic field topology, and discuss how the combined analysis of solar and interplanetary observations can be used to constrain the flare/CME ejection mechanism.
\end{abstract}

Keywords. Sun: magnetic fields, Sun: activity, Sun: flares, Sun: coronal mass ejections (CMEs)

\section{Introduction}

Solar flares and CMEs are the most violent and energetic events in the solar system. They represent the most dynamic aspect of the Sun's corona and involve a fast and large-scale destabilization of its magnetic field. As a result, large amounts of magnetic free energy contained in the coronal field is released and transformed into thermal energy, radiation, accelerated particles, and large scale flows of plasma (see Forbes 2000 for quantitative energy estimations), all of which can directly affect the Earth's environment.

Though, in general, the words "flare" or "CME" are used to refer to the radiative manifestation of energy release and the coronagraph-recorded ejection of plasma, respectively; flares and CMEs can occur separately or together in any order (Aschwanden et al. 2001), and they may, in fact, be manifestations of the same underlying physical process. In this sense, we will frequently use the term flare/CME to stress this fact. Figure 1 (top and bottom left panels) illustrates three stages of a large-scale eruptive event, observed by the Transition Region and Coronal Explorer (TRACE), during which a flare/CME occurs in close association with a filament eruption. The bottom right panel shows the chromospheric impact of energy release, but for a different event.

In this paper we will address key aspects common to flares and CMEs, such as: the origin of the energy released, the observational signatures of the energy release mechanism and the magnetic field topology where it occurs. Finally, we will comment on how we can 


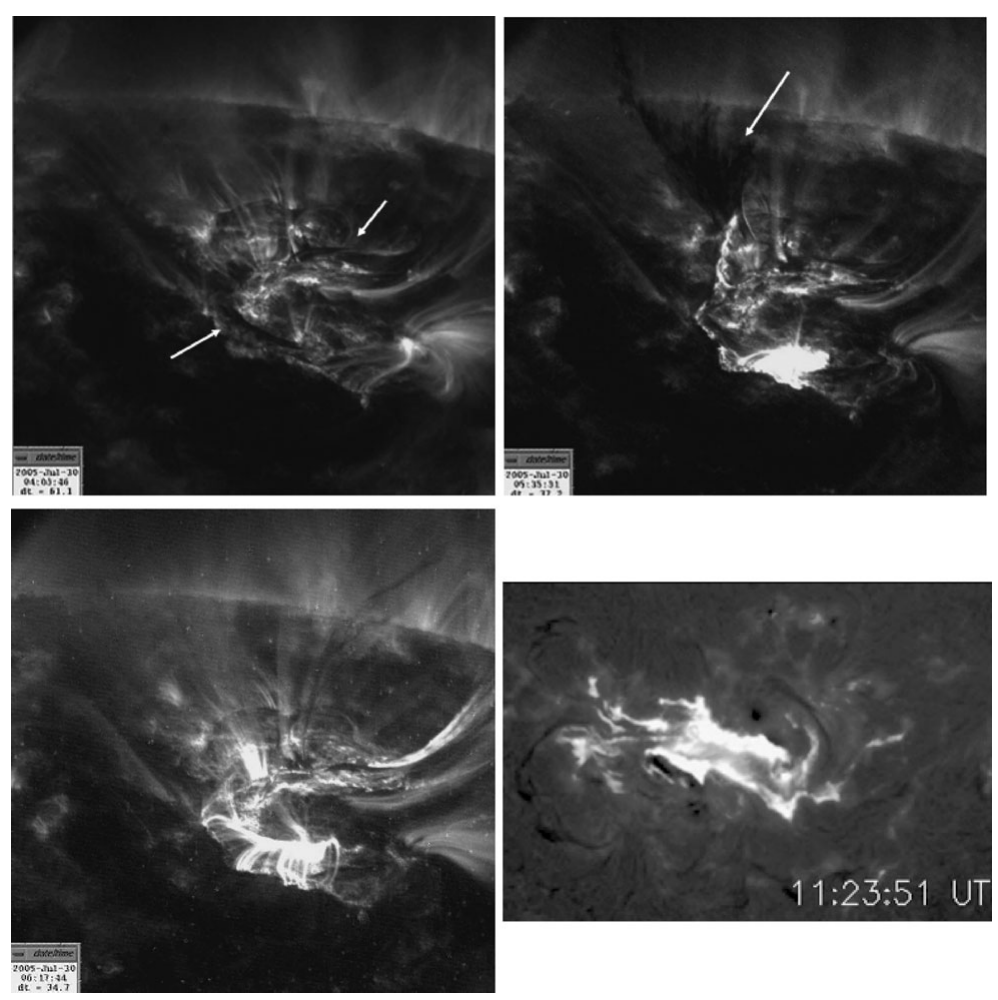

Figure 1. Top and bottom left panels show the temporal evolution of a flare/CME (TRACE, $171 \AA$ ) on 30 July 2005. The top left image (04:30 UT) shows a cool-dark curved filament (indicated by white arrows) extending along the line of sight magnetic inversion line (IL) before eruption. The top right panel (05:35 UT) depicts the filament undergoing eruption (white arrow) while flare brightenings develop at lower coronal heights. The bottom left image (06:17 UT) shows a cooling arcade of flare loops with footpoints at both sides of the IL. The bottom right panel is an $\mathrm{H} \alpha$ image taken by the $\mathrm{H} \alpha$ Solar Telescope for Argentina (HASTA) on 28 October 2003; this image shows the chromospheric response to coronal energy release during an event similar to the one in previous panels. All together, these panels illustrate the evolution of a typical two-ribbon flare.

profit from the combined study of observations from different physical regimes (i.e., the solar corona and the interplanetary (IP) space) to constrain our ideas on the flare/CME ejection mechanism. More extended reviews on flare observations and models are those by Benz (2008) and Schrijver (2009); while for CMEs, those by Schwenn (2006), Forbes (2000), Klimchuk (2001), and Forbes et al. (2006).

\section{Solar flares and coronal mass ejections: observations and models}

\subsection{General characteristics}

Energy released during large flares/CMEs can reach values in excess of $10^{33} \mathrm{erg}$. Many minor events (microflares, nanoflares) occur at energies in the range of $10^{24}-10^{25}$ erg. Historically, flares have been identified and, therefore, defined as localized brightenings observed in $\mathrm{H} \alpha$ images (e.g. Svestka 1976). These brightenings or ribbons typically appear in pairs, at both sides of the IL, and the event is called a two-ribbon flare. The standard empirical model that was built to explain the sequence of observed flare/CME manifestations is the so-called CSHKP model (Carmichael 1964, Sturrock 1966, Hirayama 


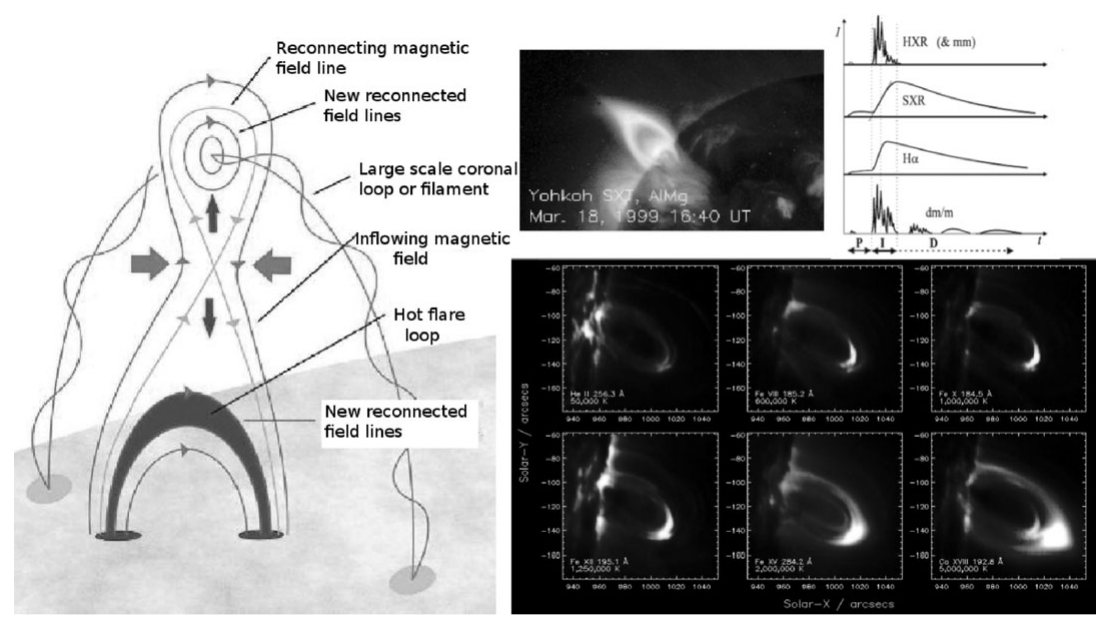

Figure 2. On the left, a cartoon representing a two-ribbon flare (adapted from Shibata 1998). On the top right, typical light-curves corresponding to emissions from the chromosphere to the corona. The image to the left is a cusp-shaped loop observed at the limb by SXT after a flare/CME on 18 March 1999 (Yokoyama et al. 2001). The bottom images show a system of loops observed at the west solar limb on 17 December 2006 by EIS. The images were taken simultaneously and show an increase with height of the loops' temperature as expected after a two-ribbon flare (figure by P. Young, see also Hara et al. 2008).

1974, Kopp \& Pneuman 1976). A cartoon of the model, typical flare light-curves, and other plasma responses to energy release are shown in Figure 2. These events occur in a dominantly bipolar magnetic configuration in which a filament, contained in a twisted flux tube, is embedded. As the configuration evolves, e.g. the shear increases close to the IL, the filament goes through a series of equilibria reaching higher coronal heights. The overlying arcade is stretched, oppositely directed field lines are brought together, and a current sheet is formed. Magnetic reconnection (see 2.3) sets in below the filament. This process implies a change of connectivity during which more flux is added to the rising filament increasing its magnetic pressure, while a lower arcade is formed below the reconnection region. As the filament continues rising, it reaches a point where no equilibrium is possible and reconnection, which is slow at first, becomes fast. The filament accelerates and erupts. Subsequent in-flowing plasma brings the surrounding field into the dissipation region and the stored energy (see 2.2) is transformed into heat and kinetic energy of accelerated particles. Large electric fields, as well as shock waves, are created in the reconnection region. Heat conduction fronts and particle beams are channeled along the newly reconnected field lines. Accelerated electrons emit gyrosynchrotron radiation as they spiral along the field lines and, as particles reach the chromosphere, hard X-rays (HXR, $\succeq 20 \mathrm{keV}$ ) are emitted by bremsstrahlung. The impulsively heated chromosphere is seen in emission in the UV and optical ranges (i.e. $\mathrm{H} \alpha$ ). The heated plasma expands upward increasing the density and temperature of the newly reconnected loops, which are observed in EUV and soft X-rays (SXR). These loops cool down and are seen later in $\mathrm{H} \alpha$. Reconnection continues between field lines which are anchored farther from the IL; newer reconnected loops are higher and the flare ribbons at their footpoints separate. Several tenths of the total flare/CME energy are released in the first few minutes during the impulsive phase. The emission in HXR, EUV, and several radio wavelengths is very structured during this phase, indicating that the energy release is intermittent. The SXR and $\mathrm{H} \alpha$ emission may rise sharply, but their maximum occurs later. During the decay phase reconnection still continues but at a decreasing rate. 

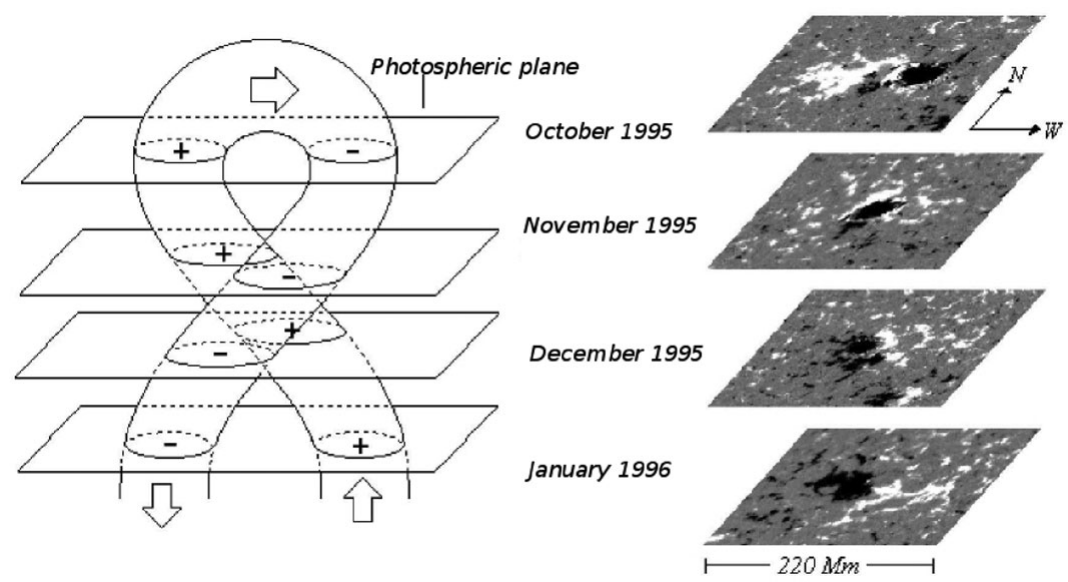

Figure 3. An example of the emergence of a twisted (kinked) flux tube. The right panel shows the photospheric evolution of AR 7912 along four rotations (line of sight magnetograms close to central meridian passage) positive/white (negative/black) values of the field are saturated above (below) 50G (-50G). On the left, we depict a sketch of the flux tube, as deduced from observations. The cuts by four horizontal planes show the approximate locations of the photosphere at the times of the magnetograms (adapted from López-Fuentes et al. 2000).

Since the CSHKP model is basically empirical, innumerable observations support the scenario; a few of the highlights are: 1) Masuda et al. (1994) observed a HXR source above a SXR loop suggesting that reconnection occurs at coronal heights. 2) Yokoyama et al. (2001) observed clear evidence of the existence of reconnection inflows in Soft X-ray Telescope (SXT) data on board Yohkoh. 3) Forbes \& Acton (1996) showed that newly formed cusped loops shrink and relax due to magnetic tension. 4) McKenzie \& Hudson (1999) observed X-ray voids in SXT and interpreted them as fast downflows due to intermittent reconnection. 5) Asai et al. (2004) showed that the start of these downflows is associated with HXR emission and microwave bursts. 5) Czaykowska et al. (1999) and Harra et al. (2005) found upflowing plasma along the outer edges of flare ribbons, while downflows were seen in the inner ribbon edges.

The events just described are associated with CMEs with cores formed by the ejected filaments. The ejected mass ranges from some $10^{13} \mathrm{~g}$ to $10^{16} \mathrm{~g}$, this plasma is embedded in a magnetic flux of $10^{20}-10^{23} \mathrm{Mx}$ (Schwenn 2006). Because the coronal pressure decreases with height, the eruptive configuration expands and, as suggested by Attrill et al. (2007) (see Mandrini et al. 2007), it may find favourably oriented surrounding fields with which reconnection takes place; then, large coronal areas may provide mass to and become part of the CME (van Driel-Gesztelyi et al. 2008).

\subsection{The origin of the energy released}

There is strong evidence showing that magnetic flux emerges twisted from the solar interior, i.e. carrying free magnetic energy to be released (Leka et al. 1996, Lites 2009). It is very difficult for a flux tube to traverse the dense photosphere, since in the concaveup parts under the flux tube axis plasma accumulates leading to its fragmentation (e.g. Manchester et al. 2004); therefore, flux tubes can only emerge through many small-scale reconnections (Pariat et al. 2004). Nevertheless, magnetic patterns, due to the contribution of the field azimuthal component, are observed as the flux tube crosses the photosphere (López-Fuentes et al. 2000); this indicates that there is an overall organization in the emerging flux which is compatible with a global twist. Furthermore, a rotation of 
the main active region (AR) polarities (see Figure 3) can be also a signature of the emergence of highly twisted flux tubes (López-Fuentes et al. 2000, 2003, Brown et al. 2003). Besides, computations of the magnetic helicity balance in ARs show that helicity has to be brought up mainly by flux emergence to explain the helicity shed via CMEs (Démoulin et al. 2002, Green et al. 2002). From a theoretical and numerical point of view, early $2 \mathrm{D}$ and 2.5D MHD simulations showed that untwisted flux tubes could not survive their transit across the convective zone since they are eroded by the vortices formed in their wake (Schüssler 1979); however, a certain amount of twist could prevent the tube fragmentation (Moreno-Insertis \& Emonet 1996). Recent 3D simulations indicate that emerging flux is less twisted than previously thought. Fan (2008) found that to comply with Joy's law, the twist-induced tilt in rising flux ropes should be less than half of that needed for a cohesive rise. These simulations and observations imply that all the large-scale flux that has crossed the convective zone must be twisted.

Before the finding that flux emerges twisted, the generally accepted idea was that free magnetic energy is built up by shearing motions. Computations were done to estimate the time these motions should be imposed to store enough energy to power a flare (see e.g McClymont \& Fisher 1989). Large-scale flows are present in the solar photosphere (e.g. differential rotation) and local deviations from the mean differential rotation rate are seen in flow-maps of the solar surface (e.g. Meunier 2005). Leka et al. (1996) clearly showed, however, that shearing footpoint motions were insufficient to create all the electric currents observed in an emerging AR. Furthermore, apparent surface shearing flows can result from the emergence of a flux tube (Démoulin \& Berger 2003). In addition to organized patterns, random footpoint motions are also present.

\subsection{The energy release mechanism and the field topology}

Magnetic field reconnection, that can be defined as a topological restructuring of the field which leads to a change of its connectivity, has been the energy release mechanism proposed at the origin of flare and some CME models. From a theoretical point of view, magnetic configurations with a complex topology, i.e. having separatrices (places where the field connectivity is discontinuous), are the locations where current sheets can form in $2 \mathrm{D}$. When going to 3D, and if the photospheric magnetic field is described by a series of isolated polarities (surrounded by field-free regions), a complete topological description of the magnetic configuration is given by the skeleton formed by null points, spines, fans, separators, and associated separatrices (Longcope 2005 and examples therein).

However, if the photosphere is fully magnetized, most of the above topological structures disappear; only separatrices associated to coronal magnetic nulls remain. Separatrices of a different origin are linked to concave-up field lines tangential to the photosphere (defining bald-patch locations) (Titov et al. 1993). For some observed configurations, those structures are sufficient to explain the location of flare kernels resulting from reconnection. Examples have been found where coronal null points, computed using either subphotospheric sources or extrapolations to represent the coronal field, were associated with coronal activity (Mandrini et al. 1991, Gaizauskas et al. 1998, Aulanier et al. 2000, Fletcher et al. 2001a, Mandrini et al. 2006, Luoni et al. 2007). Concerning bald patches, they have been only found in connection with low energy release events: small flares (Aulanier et al. 1998), EUV brightenings (Fletcher et al. 2001b), surges (Mandrini et al. 2002), and Ellerman bombs (Pariat et al. 2004).

The analysis of the topological structure of numerous ARs has shown that flares occur in a variety of configurations larger than just discussed (see e.g. Démoulin et al. 1994 and the reviews by Démoulin 2006, 2007). Quasi-separatrix layers (QSLs) (Priest \& Démoulin 1995, Démoulin et al. 1996, Titov et al. 2002), which are regions where there is a 

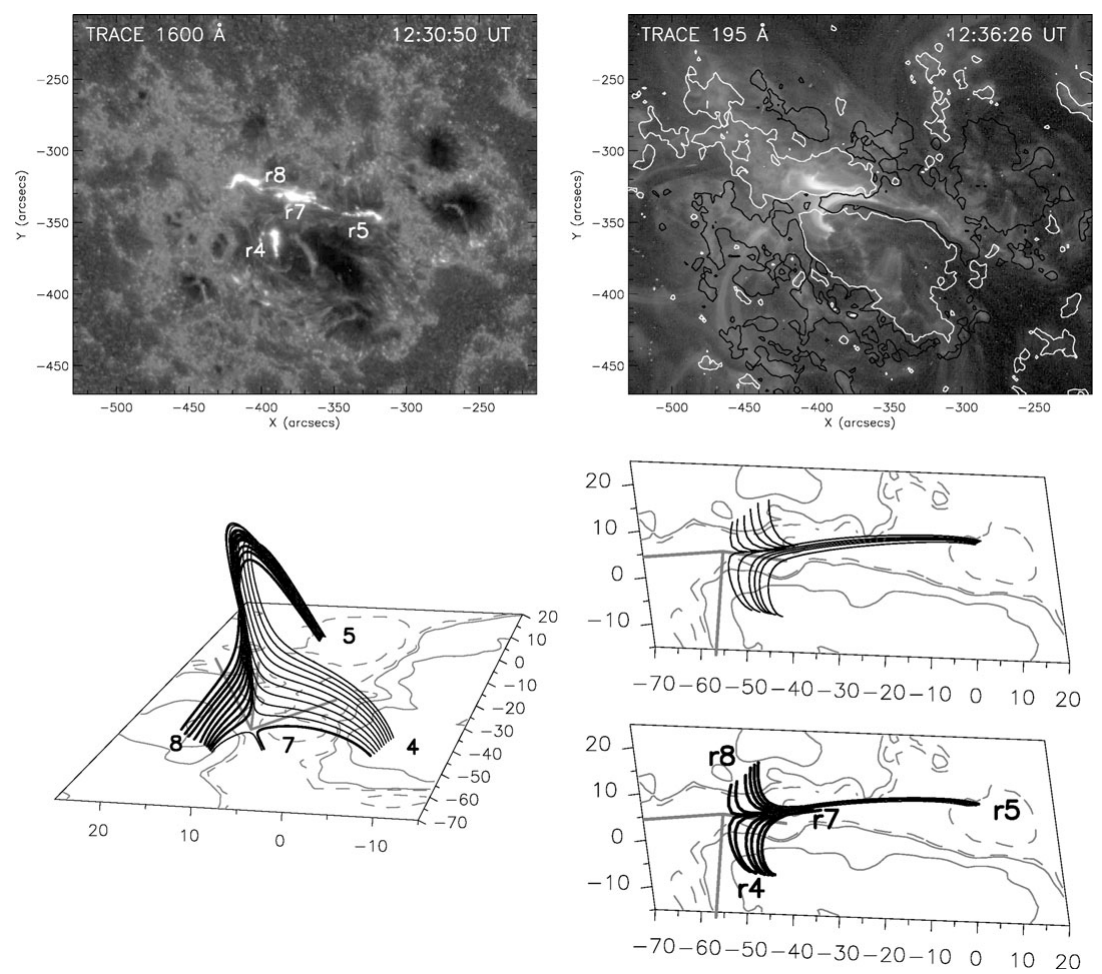

Figure 4. Top panels: TRACE images of AR 10486 on 27 October, 2003, (1600 $\AA$ (left) and $195 \AA$ (right)) showing the locations of flare kernels (r4, r5, r7 and r8). The bottom left panel depicts the location of a 3D coronal null point (three lines) with field lines computed starting integration at finite distances from the null. A set of thin continuous lines have footpoints in polarities 4 and 5 . These lines could reconnect at the null with lines linking 7 to 8 , represented by only one short and thin continuous line. Reconnection forms the set of thick continuous field lines with footpoints at 8 and 5 , and those that connect 4 and 7 (the latter are represented by only one thick line). The two bottom-right panels show the coronal field model in the vicinity of the null point. Field lines are the same as in the left panel but as seen from the observer's point of view. The upper of these panels shows two sets of lines representing the pre-reconnected loops. The lower one corresponds to field lines after reconnection (notice the similarity between their shape and loops in $195 \AA$ ). Several short field lines have been added as compared to the left panel. Negative (positive) field isocontours are shown in continuous (dashed) thin lines, their values are \pm 100 and $\pm 1000 \mathrm{G}$. The axes are labeled in Mm (adapted from Luoni et al. 2007).

drastic change in field-line linkage, generalize the concept of separatrices to configurations without null points and/or bald patches. Using coronal field models, QSLs have been found to exist in the broadest variety of observed magnetic configurations (Démoulin et al. 1997, Fletcher et al. 2001b, Bagalá et al. 2000). QSLs have been shown to coincide with flare kernels in the chromosphere and coronal loop brightenings of various intensity. These brightenings were connected by field lines as expected from the magnetic reconnection theory. Moreover, in cases where vector magnetograms were available, photospheric current concentrations were also located at the photospheric trace of QSLs. Recently, to relate QSLs with the formation of strong current concentrations and study the characteristics of the reconnection process, 3D MHD simulations of typical observed field distributions and photospheric motions have been developed by Aulanier et al. (2005, 2006). The results of these simulations imply that electric currents at QSLs may amplify in time only if the QSLs are broader than the dissipative scale length, as it was suggested in previous observational studies. Related to magnetic reconnection, the simulations show 

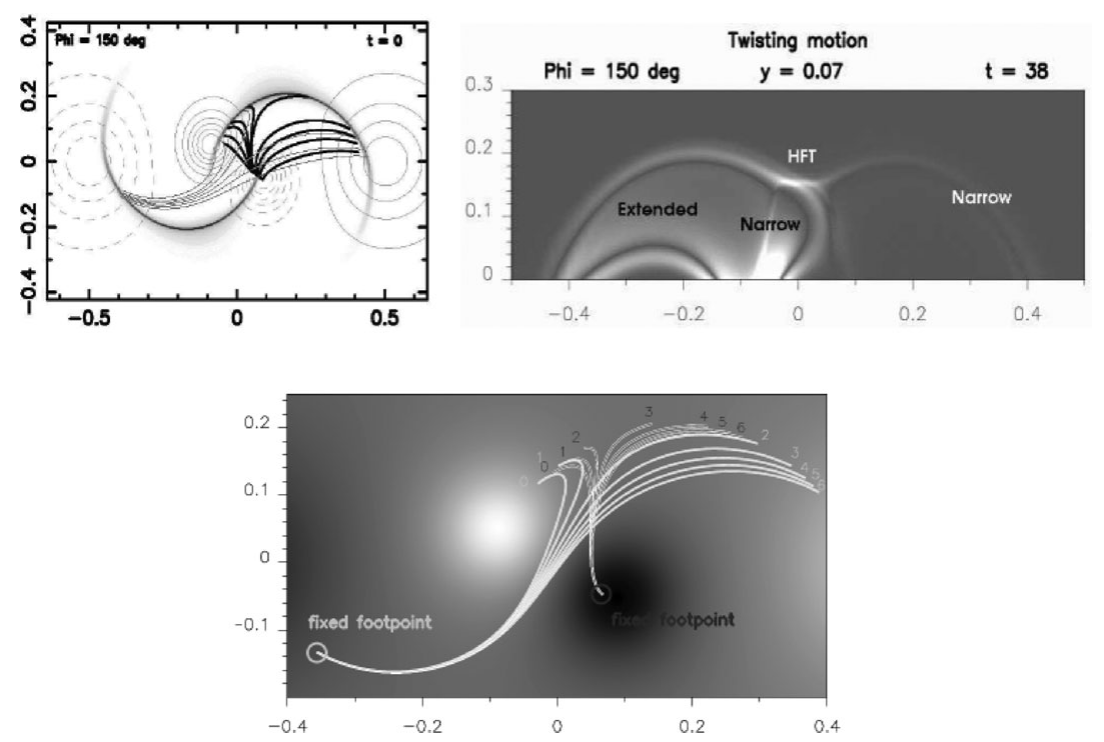

Figure 5. Top left panel: Location of QSLs in a simple magnetic configuration formed by two magnetic bipoles. Two sets of field lines are added to illustrate the drastic change in connectivity, both sets have footpoints starting along a segment crossing a QSL at the lower boundary. Continuous (dashed) contours show positive (negative) values of the vertical field component. Top right panel: Formation of strong electric currents at QSLs after the configuration on the left has evolved in a MHD simulation (twisting motions of the smallest positive polarity). The figure is a vertical cut of the $3 \mathrm{D}$ configuration along a horizontal line at $\mathrm{y}=0.07$ in the top left panel. The current scales linearly with the grey-scale intensity (white is the highest value). Extended and narrow currents layers coexist. Bottom panel: Magnetic reconnection after the same twisting motions are applied. The evolution of two slip-running field lines with fixed footpoints in the negative polarities is shown with a fixed time step; as reconnection proceeds, each line changes its connectivity, shifting along the QSL. Figures are adapted from Aulanier et al. $(2005,2006)$.

that it occurs only due to the self-pinching and dissipation of narrow current layers previously formed at QSLs. A property of reconnection is the continuous slippage of field lines along each other as they pass through the current layers. Observational evidence of this slip-running reconnection has been found in flares seen by the X-ray Telescope on board Hinode (Aulanier et al. 2007) and by TRACE (Masson et al. 2009). The events analyzed in the papers mentioned previously are confined flares, the connectivity change occurs between two closed field configurations, since this is linked to the intrinsic definition of QSLs. However, energy release by reconnection may occur in more general topologies (see 2.1). QSLs have been recently found associated with intense outflows observed by the Extreme-ultraviolet Imaging Spectrometer (EIS) on board Hinode at the border of ARs (between closed and large-scale open-like field lines) by Baker et al. (2009), indicating that signatures of energy release along QSLs is even broader. Figures 4 and 5 show the topology of an observed AR and a MHD simulation.

\subsection{CMEs initiation models and the link to interplanetary events}

CME initiation models can be divided in different ways, i.e. taking into account the presence of non-ideal or ideal instabilities as the drivers (Forbes 2000), or in a tutorial way emphasizing their basic physical differences (Klimchuk 2001). We will follow a different approach and will separate models according to characteristics that may be useful when comparing solar to IP associated events; that is, models in which the ejected flux tube is mainly formed during the eruption from models in which it is already present in the pre-eruptive configuration. Furthermore, it is not our intention to make a detailed 
description of any of these models. In the first group, we put two sets of models: a) the classical tether cutting and flux cancellation models (Moore \& Rumeolitis 1992, Moore et al. 2001, Mikic \& Linker 1994), and b) the breakout model (Antiochos et al. 1999, Lynch et al., 2004, 2008). This group of models requires the presence of sheared arcades and reconnection to start the eruption. The fundamental difference between both sets is that reconnection initially occurs in the core sheared field in the former and above it in the breakout model. Therefore, the latter requires the presence of a null point and associated separatrices or, in a more general way, QSLs in the corona; the basic magnetic field configuration is quadrupolar. In the first set the flux tube forms from the initial arcade reconnection process; in the second set, the flux tube forms when reconnection below the rising arcade sets in. For the second group of models, the eruption is driven by an ideal MHD process: a) a catastrophe (Forbes \& Isenberg 1991, Isenberg et al. 1993, Lin et al. 1998), or b) an instability (kink and/or torus instabilities) (Fan \& Gibson 2003, 2004, 2007, Török \& Kliem 2003, 2005, Kliem et al. 2004, Török et al. 2004, Kliem \& Torok 2006). Reconnection is not required initially but may help to trigger the eruption.

Following a flare/CME, plasma and magnetic field expand into the IP space. The resulting structures are called interplanetary CMEs (ICMEs). The term magnetic cloud (MC), where more restricted characteristics are present, is used for a subset of ICMEs. We can relate $\mathrm{MC}$ physical parameters to those of the solar eruptive region; a relevant one is the magnetic flux, since the magnetic flux involved in the eruption should equate the flux in the MC. On the Sun, surface phenomena, such as coronal dimmings, can be identified and associated with the footpoints of the erupting structure. Since the usual interpretation of dimmings is that they are the sites of material outflow (e.g. Harra \& Sterling 2001) and two dimmings are often located on both sides of the eruption site over opposite magnetic polarities, it has frequently been assumed (Webb et al. 2000) that the dimmings form at the ejected flux tube footpoints. Magnetic flux in the dimmings may be measured from magnetograms and compared with the flux in the related MC. Axial and azimuthal fluxes are computed from in situ magnetic data by fitting a model (Dasso et al. 2005). Comparisons often show rough agreements between flux in dimmings and cloud axial fluxes (Lepping et al. 1997, Webb et al. 2000). However, MCs are highly twisted structures and may have substantial azimuthal flux. Mandrini et al. (2005) and Attrill et al. (2006) have found an excellent agreement between flux in dimmings and the azimuthal MC flux. Twist and flux (in an overlying arcade) may be added via reconnection to an expanding tube, such that the flux from dimmings can contribute to the cloud azimuthal flux component; therefore, these results (see also Qiu et al. 2007) suggest that the ejected flux tube is mainly formed during the eruption.

\section{Discussion and conclusions}

From this concise overview of flare/CME characteristics, we can conclude that the magnetic free energy, which is released during these events, is mostly brought up from the solar interior by twisted flux emergence. Though all observational evidence is indirect, there is a consensus that the energy release mechanism at work is magnetic reconnection. This is at the origin of flares and CMEs, and it even contributes to the acceleration of the eruption in some cases. The observational examples showing various levels of activity originating from different topologies, teach us that reconnection can occur in configurations with much wider topological characteristics than was traditionally considered. QSLs, a generalization of separatrices to configurations in which the magnetic connectivity is not discontinuous (but changes drastically), are the most common locations where reconnection takes place. Three-dimensional MHD simulations have shown that magnetic reconnection proceeds in such way that field lines slip-run along 
QSLs as they reconnect. Combined quantitative studies of solar events and their IP counterparts can greatly contribute to our understanding of the flare/CME eruption mechanism. The few examples analyzed so far indicate that the flux tube observed in the IP space is mainly formed during the eruption.

To further understand the details of solar activity events (flares/CMEs) from the buildup of magnetic energy to its effective release and evolution in the IP space, we need both high-resolution measurements of all three components of the magnetic field vector at different solar atmospheric heights, improved 3D views (measurements) of the events from the Sun through the IP space (as the STEREO spacecraft are providing), as well as the development of more realistic 3D models of the coronal and IP magnetic field. Obvious though these assertions may sound, we all know that they are not easy to achieve.

\section{References}

Asai, A., Yokoyama, T., Shimojo, M., \& Shibata, K. 2004, ApJ, 605, L77

Attrill, G. D. R., Harra, L. K., van Driel-Gesztelyi, L., \& Démoulin, P. 2007, ApJ, 656, L101

Attrill, G., Nakwacki, M. S., \& Harra, L. K., et al. 2006, Sol. Phys., 238, 117

Aulanier, G., et al. 2007, Science, 318, 1588

Aulanier, G., DeLuca, E. E., Antiochos, S. K., McMullen, R. A., \& Golub, L. 2000, ApJ, 540, 1126

Aulanier, G., Démoulin, P., Schmieder, B., Fang, C. \& Tang, Y. H. 1998, A\&A, 183, 369

Aulanier, G., Pariat, E., \& Démoulin, P. 2005, A\& A, 444, 961

Aulanier, G., Pariat, E., Démoulin, P., \& Devore, C. R. 2006, Sol. Phys., 238, 347

Antiochos, S. K., DeVore, C. R., \& Klimchuk, J. A. 1999, ApJ, 510, 485

Aschwanden, M. J., Poland, A. I., \& Rabin, D. M. 2001, Ann. Rev. Astron. Astrophys., 39, 175

Bagalá, L. G., Mandrini, C. H., Rovira, M. G., \& Démoulin, P. 2000, A\&A, 363, 779

Baker, D., van Driel-Gesztelyi, L., Mandrini, C. H., Démoulin, P., et al. 2009, ApJ, 705, 926

Benz, A. O. 2008, Liv. Rev. Sol. Phys., 5, 1

Brown, D. S., Nightingale, R. W., Alexander, D., Schrijver, C. J., et al. 2003, Sol. Phys., 216, 79

Carmichael, H. 1964, NASA SP, 50, 451

Czaykowska, A., de Pontieu, B., Alexander, D., \& Rank, G. 1999, ApJ, 521, L75

Dasso, S., Mandrini, C. H., Démoulin, P., Luoni, M. L., et al. 2005, Adv. Spa. Res., 35, 711

Démoulin, P. 2006, Adv. Spa. Res., 37, 1269

Démoulin, P. 2007, Adv. Spa. Res., 39, 1367

Démoulin, P., Bagalá, L. G., Mandrini, C. H., Hénoux, J. C., \& Rovira, M. G. 1997, A\&A, 325, 305

Démoulin, P. \& Berger, M. A. 2003, Sol. Phys., 215, 203

Démoulin, P., Hénoux, J. C., \& Mandrini, C. H. 1994, A\&\&A, 285, 1023

Démoulin, P., Hénoux, J. C., Priest, E. R., Mandrini, C. H. 1996, A\& A, 308, 643

Démoulin, P., Mandrini, C. H., \& van Driel-Gesztelyi, L., et al. 2002, A\&A, 382, 650

Fan, Y. 2008, ApJ, 676, 680

Fan, Y. \& Gibson, S. E. 2003, ApJ, 589, L105

Fan, Y. \& Gibson, S. E. 2004, ApJ, 609, 1123

Fan, Y. \& Gibson, S. E. 2007, ApJ, 668, 1232

Fletcher, L., López Fuentes, M. C., Mandrini, C. H., et al. 2001b, Solar Phys., 203, 255

Fletcher, L., Metcalf, T. R., Alexander, D., Brown, D. S., \& Ryder, L. A. 2001a, ApJ, 554, 451

Forbes, T. G. 2000, JGR, 105, 23153

Forbes, T. G. \& Acton, L. W. 1996, ApJ, 459, 330

Forbes, T. G. \& Isenberg, P. A. 1991, ApJ, 373, 294

Forbes, T. G., Linker, J. A., Chen, J., Cid, C., Kóta, J., et al. 2006, Spa. Scien. Rev., 123, 251

Gaizauskas, V., Mandrini, C. H., Démoulin, P., Luoni, M. L., \& Rovira, M. G. 1998, A\&3A 332, 353

Hara, H., Watanabe, T., Matsuzaki, K., \& Harra, L. K., et al. 2008, PASJ, 60, 275 
Harra, L. K., Démoulin, P., Mandrini, C. H., Matthews, S. A., et al. 2005, A\&̋A, 438, 1099

Harra, L. K. \& Sterling, A. C. 2001, ApJ, 561, L215

Hirayama, T. 1974, Sol. Phys., 34, 323

Isenberg, P. A., Forbes, T. G., \& Démoulin, P. 1993, ApJ, 417, 368

Kliem, B., Titov, V. S., \& Török, T. 2004, A\&AA, 413, L23

Kliem, B. \& Torok, T. 2006, PRL, 96, 255002

Klimchuk, J. A. 2001, in: P. Song, H. Singer \& G. Siscoe (eds.), Geophys. Mon., 125, 143, AGU

Kopp, R. A. \& Pneuman, G. W. 1976, Sol. Phys., 50, 85

Leka, K. D., Canfield, R. C., McClymont, A. N., \& van Driel-Gesztelyi, L. 1996, ApJ, 462, 547

Lepping, R. P., Szabo, A., DeForest, C. E., \& Thompson, B. J. 1997, ESA SP, 415, 163

Lin, J., Forbes, T. G., Isenberg, P. A., \& Démoulin, P. 1998, ApJ, 504, 1006

Lites, B. W. 2009, Spa. Sci. Rev., 144, 197

López Fuentes, M. C., Demoulin, P., Mandrini, C. H., \& van Driel-Gesztelyi, L. 2000, ApJ, 544, 540

López Fuentes, M. C., Démoulin, P., Mandrini, C. H., et al. 2003, A\& $\&$ A, 397, 305

Longcope, D. W. 2005, Liv. Rev. Sol. Phys. 2, 7

Luoni, M. L., Mandrini, C. H., Cristiani, G. D., \& Démoulin, P. 2007, Adv. Spa. Res., 39, 1382

Lynch, B. J., Antiochos, S. K., DeVore, C. R., Luhmann, J. G., et al. 2008, ApJ, 683, 1192

Lynch, B. J., Antiochos, S. K., MacNeice, P. J., Zurbuchen, T. H., \& Fisk, L. A. 2004, ApJ, 617,589

Manchester, W., IV, Gombosi, T., DeZeeuw, D., \& Fan, Y. 2004, ApJ, 610, 588

Mandrini, C. H., Démoulin, P., Hénoux, J.-C., \& Machado, M. E. 1991, A\& A 250, 541

Mandrini, C. H., Demoulin, P., Schmieder, B., Deluca, E. E., et al. 2006, Sol. Phys., 238, 293

Mandrini, C. H., Démoulin, P., Schmieder, B., Deng, Y. Y., \& Rudawy, P. 2002, A\& A, 391, 317

Mandrini, C. H., Nakwacki, M. S., Attrill, G., et al. 2007, Sol. Phys., 244, 25

Mandrini, C. H., Pohjolainen, S., Dasso, S., Green, L. M., et al. 2005, A\& A, 434, 725

McClymont, A. N. \& Fisher, G. H. 1989, in: J. Waite, J. Burch, \& R. Moore (eds.), Solar System Plasma Physics, 219, AGU

McKenzie, D. E. \& Hudson, H. S. 1999, ApJ, 519, L93

Masson, S., Pariat, E., Aulanier, G., \& Schrijver, C. J. 2009, ApJ, 700, 559

Masuda, S., Kosugi, T., Hara, H., Tsuneta, S., \& Ogawara, Y. 1994, Nature, 371, 495

Meunier, N. 2005, A\&A, 443, 309

Mikic, Z. \& Linker, J. A. 1994, ApJ, 430, 898

Moreno-Insertis, F. \& Emonet, T. 1996, ApJ, 472, L53

Moore, R. L. \& Roumeliotis, G. 1992, IAU Colloq. 133: Eruptive Solar Flares, 399, 69

Moore, R. L., Sterling, A. C., Hudson, H. S., \& Lemen, J. R. 2001, ApJ, 552, 833

Pariat, E., Aulanier, G., Schmieder, B., Georgoulis, M. K., et al. 2004, A\&\&A 614, 1099

Priest, E. R. \& Démoulin, P. 1995, JGR, 100, 23443

Qiu, J., Hu, Q., Howard, T. A., \& Yurchyshyn, V. B. 2007, ApJ, 659, 758

Schrijver, C. J. 2009, Adv. Spa. Res., 43, 739

Schrijver, C. J., De Rosa, M. L., Metcalf, T., Barnes, G., et al. 2008, Astrophys. J., 675, 1637

Schwenn, R. 2006, Liv. Rev. Sol. Phys., 3, 2

Shibata, K. 1998, Ap\&SSS, 264, 129

Sturrock, P. A. 1966, Nature, 211, 695

Schüssler, M. 1979, A\&SA, 71, 79

Svestka, Z. 1976, Solar Flares, Springer-Verlag Berlin Heidelberg

Titov, V. S., Priest, E. R., \& Démoulin, P. 1993, A\&A, 276, 564

Titov, V. S., Hornig, G., \& Démoulin, P. 2002, JGR, 107, SSH 3, 1-13

Török, T. \& Kliem, B. 2003, A\&GA, 406, 1043

Török, T. \& Kliem, B. 2005, ApJ, 630, L97

Török, T., Kliem, B., \& Titov, V. S. 2004, A\&AA, 413, L27

van Driel-Gesztelyi, L., Attrill, G., Démoulin, P., Mandrini, C., et al. 2008, An. Geo., 26, 3077

Webb, D. F., Lepping, R. P., Burlaga, L. F., DeForest, C. E., et al. 2000, JGR, 105, 27251

Yokoyama, T., Akita, K., Morimoto, T., Inoue, K., \& Newmark, J. 2001, ApJ, 546, L69 